\title{
A C-BAND CROSS POLARIZATION GEOPHYSICAL MODEL FUNCTION
}

\author{
Paul A. Hwang ${ }^{1}$, Ad Stoffelen ${ }^{2}$, Gerd-Jan van Zadelhoff², William Perrie ${ }^{3}$, Biao Zhang ${ }^{3,4}$, Haiyan Li ${ }^{3,5}$, and Hui Shen ${ }^{3,6}$ \\ ${ }^{1}$ Remote Sensing Division, Naval Research Laboratory, Washington DC, USA \\ ${ }^{2}$ Royal Netherlands Meteorological Institute, De Bilt, the Netherlands \\ ${ }^{3}$ Fisheries and Oceans Canada, Bedford Institute of Oceanography, Dartmouth, Nova Scotia, Canada \\ ${ }^{4}$ School of Marine Sciences, Nanjing University of Information Science and Technology Nanjing, Nanjing, China \\ ${ }^{5}$ Key Laboratory of Computational Geodynamics, Chinese Academy of Sciences and University of Chinese Academy \\ of Sciences, Beijing, China \\ ${ }^{6}$ Institute of Oceanology, Chinese Academy of Sciences, Qingdao, China
}

\section{INTRODUCTION}

Microwave backscattering from the ocean surface is closely related to the wind-generated ocean surface roughness. This property is used for obtaining global ocean surface vector winds. The deployed scatterometers so far do not use the cross-polarized sea return ( $\mathrm{VH}$, representing either vertical transmit horizontal receive or horizontal transmit vertical receive) because of its weak signal level. The copolarized returns ( $V V$ or $H H$ ), however, may saturate in high wind speeds especially for low incidence angles.

Recent analyses suggest that the saturation of C-band $V H$ signal, if it exists, may occur at much higher wind velocities in comparison to the co-polarized signals (Hwang et al., 2010a, b; Vachon and Wolfe, 2011; Zhang et al., 2011). The result prompts the incorporation of $V H$ channel in the next generation scatterometer designs for extending the retrieved wind speed range (Lin et al. 2012; Belmonte Rivas et al., 2014). In the meantime, much effort is devoted to developing cross polarization geophysical model functions (GMFs) for hurricane wind retrieval using polarimetric SAR imagery. Several of the GMFs assume a linear relationship between $V H$ in $\mathrm{dB}$ and wind speed $U_{10}$ (Zhang et al., 2014; van Zadelhoff et al., 2013, 2014). One
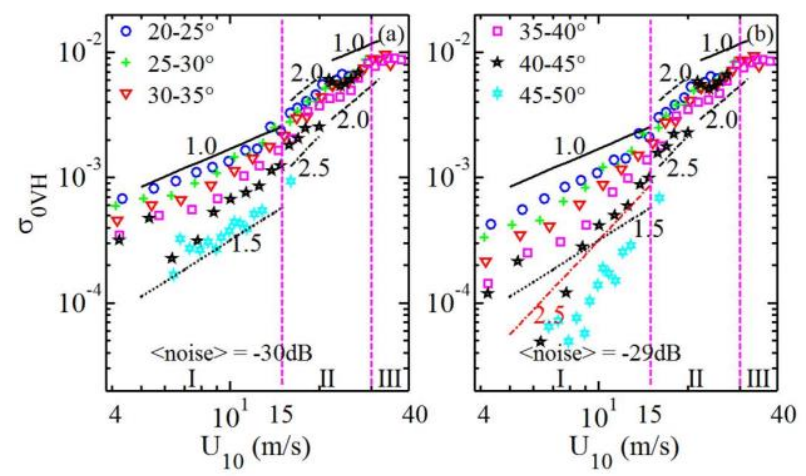

Fig. 1. Piecewise power law relationship between $V H$ NRCS and wind speed illustrated by the BHS dataset: (a) assuming $-30 \mathrm{~dB}$ average noise level, and (b). assuming -29 dB average noise level. drawback of these GMFs is the lack of incidence angle $(\theta)$ dependence, at least for the high wind range $\left(U_{10}>21 \mathrm{~m} / \mathrm{s}\right)$.

As illustrated in Fig. 1, recent analyses show that the dependence of $V H$ in linear scale on $U_{10}$ can be expressed in piecewise power-law functions (Hwang et al., 2014, 2015). Here, we present a C-band GMF using the piecewise powerlaw relationship. Presented in this study are three datasets (Zhang et al., 2014; van Zadelhoff et al., 2013, 2014) composed of R2 dual-polarization ( $V V$ and $V H$ ) NRCS and: (1) wind data from Buoy, stepped frequency microwave radiometer (SFMR) and $\mathrm{H}^{*} \mathrm{~W}$ ind sources: $\mathrm{BSH}$; (2) wind data from SFMR measurements: $\mathrm{KNMI}_{\mathrm{S}}$; and (3) wind data from ECMWF models: $\mathrm{KNMI}_{\mathrm{E}}$. The proposed GMF incorporates the incidence angle dependence for the full range of wind speed available in our datasets (up to $56 \mathrm{~m} / \mathrm{s}$ ).

\section{THE GMF DESIGN}

The proposed GMF is designed by representing the $V H$ as power-law functions of wind speed in several wind speed groups (WSGs), with the proportionality coefficient and the exponent of the power-law function varying with the incidence angle. For the $n$-th WSG, this is written as

$$
\left[\sigma_{0 V H}(\theta)\right]_{n}=A_{n}(\theta) U_{10}^{a_{n}(\theta)} .
$$

In order to adapt the better information in the future, easy modification of the GMF is an important consideration. For example, the number of WSGs and the parameterization in each WSG can be revised without major changes in the design structure as the database expands and knowledge of wind speed dependence in each WSG and incidence angle bin (IAB) becomes more refined.

Ideally, the coefficient $A_{n}$ and exponent $a_{n}$ can be derived from field data. Presently, only partial information is available or can be estimated due to a lack of comprehensive coverage in $\theta$ and $U_{10}$ in our collected datasets. Some of the parameters can be computed with the requirement of continuity between neighboring WSGs of a given $\theta$. In particular, if only the exponents $a_{n}$ for all WSGs and a limited number of $A_{n}$ can be specified, the remaining proportionality coefficients $A_{n}$ can be obtained by matching 
Table 1. C-Band VH GMF Parameters.

\section{a. BSH \& KNMIs}

$\begin{array}{lcrrrrrrrrr}\theta & A_{1} & a_{1} & U_{t 1} & a_{2} & U_{t 2} & a_{3} & U_{t 3} & a_{4} & U_{t 4} & a_{5} \\ 17.5 & 1.40 \mathrm{E}-04 & 0.90 & 10.00 & 2.00 & 21.00 & 1.10 & 25.00 & 0.75 & 30.00 & -0.25 \\ 22.5 & 9.06 \mathrm{E}-05 & 1.10 & 11.00 & 2.25 & 21.00 & 1.10 & 25.00 & 0.75 & 33.00 & -0.25 \\ 27.5 & 5.33 \mathrm{E}-05 & 1.30 & 12.00 & 2.35 & 21.00 & 1.50 & 32.00 & 0.75 & 35.00 & -0.25 \\ 32.5 & 2.79 \mathrm{E}-05 & 1.50 & 14.00 & 2.50 & 21.00 & 1.50 & 34.00 & 1.00 & 35.00 & -0.25 \\ 37.5 & 1.34 \mathrm{E}-05 & 1.70 & 15.00 & 2.70 & 21.00 & 2.00 & 34.00 & 1.50 & 35.00 & -0.25 \\ 42.5 & 5.44 \mathrm{E}-06 & 1.90 & 15.00 & 3.00 & 21.00 & 2.60 & 28.00 & 1.00 & 40.00 & -0.50 \\ 47.5 & 1.15 \mathrm{E}-06 & 2.10 & 15.00 & 3.60 & 21.00 & 3.50 & 28.00 & 3.00 & 50.00 & 1.50 \\ 52.5 & 8.00 \mathrm{E}-07 & 2.30 & 15.00 & 3.60 & 21.00 & 3.50 & 28.00 & 3.00 & 50.00 & 1.50\end{array}$

\section{b. KNMI $_{\mathrm{E}}$}

$\begin{array}{lllllllllll}17.5 & 1.40 \mathrm{E}-04 & 0.90 & 10.00 & 2.00 & 21.00 & 1.50 & 28.00 & 0.75 & 30.00 & 0.75 \\ 22.5 & 9.06 \mathrm{E}-05 & 1.10 & 11.00 & 2.25 & 21.00 & 1.50 & 32.00 & 1.00 & 33.00 & 1.00 \\ 27.5 & 5.33 \mathrm{E}-05 & 1.30 & 12.00 & 2.35 & 21.00 & 2.00 & 32.00 & 1.00 & 40.00 & 1.00 \\ 32.5 & 2.79 \mathrm{E}-05 & 1.50 & 14.00 & 2.50 & 21.00 & 2.00 & 34.00 & 1.00 & 40.00 & 1.00 \\ 37.5 & 1.34 \mathrm{E}-05 & 1.70 & 15.00 & 3.00 & 21.00 & 2.00 & 34.00 & 1.20 & 40.00 & 1.20 \\ 42.5 & 8.16 \mathrm{E}-06 & 1.90 & 15.00 & 3.00 & 21.00 & 2.00 & 28.00 & 1.20 & 40.00 & 1.20 \\ 47.5 & 3.45 \mathrm{E}-06 & 2.10 & 15.00 & 3.50 & 21.00 & 1.50 & 28.00 & 1.50 & 50.00 & 1.50 \\ 52.5 & 8.00 \mathrm{E}-07 & 2.30 & 15.00 & 3.20 & 21.00 & 1.50 & 28.00 & 1.50 & 50.00 & 1.50\end{array}$

the $\sigma_{0 V H}$ of neighboring WSGs at the corresponding transition wind speed $U_{t}$. This leads to a recursive equation

$$
A_{n}(\theta)=A_{n-1}(\theta) U_{t(n-1)}^{a_{n-1}(\theta)-a_{n}(\theta)},
$$

where $U_{t(n-1)}$ is the transition velocity between $(n-1)$-th and $n$-th WSGs. We can expect to have the most abundant observations in WSG1 for producing $A_{1}$ and $a_{1}$ in all $\theta$ bins. It becomes clear that only a reasonable knowledge of the wind speed exponents in higher wind speed groups is needed for completing the $V H$ GMF formulation. The procedure is summarized in the following steps:

Step 1: Divide data into some finite number of IABs.

Step 2: For each $i$-th IAB determine the number of WSGs and estimate the appropriate $a_{1}\left(\theta_{i}\right)$, $U_{t 1}\left(\theta_{i}\right), a_{2}\left(\theta_{i}\right), U_{t 2}\left(\theta_{i}\right), a_{3}\left(\theta_{i}\right), U_{t 3}\left(\theta_{i}\right), a_{4}\left(\theta_{i}\right), \ldots$

Step 3: Use polynomial data fitting and obtain $A_{1}\left(\theta_{i}\right)$ for each $i$-th IAB in WSG1.

Step 4: Compute $A_{2}\left(\theta_{i}\right), A_{3}\left(\theta_{i}\right), \ldots$ using (2);

Step 5: Interpolate $A_{n}(\theta)$ and $a_{n}(\theta)$ using the corresponding bin values $A_{n}\left(\theta_{i}\right)$ and $a_{n}\left(\theta_{i}\right)$.
Step 6: Compute $\sigma_{0 \mathrm{VH}}\left(\theta ; U_{10}\right)$ with (1) using the $A_{n}(\theta)$ and $a_{n}(\theta)$ determined in steps 1 to 5 .

From examining the available data, it is judged that 5 WSGs can provide sufficient description of the available data with wind speed coverage up to $56 \mathrm{~m} / \mathrm{s}$. The recommended $A_{1}\left(\theta_{i}\right), a_{n}\left(\theta_{i}\right)$ and $U_{t n}\left(\theta_{i}\right)$ are listed in Table 1a. To serve as a lookup table (LUT) for $\theta$ interpolation over the range between $20^{\circ}$ and $50^{\circ}$, two additional entries are added for $\theta$ bins $15^{\circ}-20^{\circ}$ and $50^{\circ}-55^{\circ}$ by extrapolation. The $\mathrm{KNMI}_{\mathrm{E}}$ dataset differs substantially from BSH and KNMI . Adjustments for the differences in $A_{1}\left(\theta_{i}\right), U_{t n}\left(\theta_{i}\right)$ and $a_{n}\left(\theta_{i}\right)$ are listed in Table $1 b$.

Figure 2 shows a comparison of the proposed GMF with three recently published $V H$ GMFs, referred to as vZ13s, vZ13 $\mathrm{E}$ (van Zadelhoff et al., 2013, 2014) and Z14 (Zhang et al., 2014). In the same convention, the GMFs described in this paper are called $\mathrm{H} 14_{\mathrm{S}}$ and $\mathrm{H} 14_{\mathrm{E}}$. To reduce clutter, we only show data in $3 \theta$ bins: $22.5^{\circ} \pm 2.5^{\circ}$, $37.5^{\circ} \pm 2.5^{\circ}$ and $47.5^{\circ} \pm 2.5^{\circ}$. The BSH (dark symbols) and KNMI (light symbols) data are combined in Fig. 2a and the $\mathrm{KNMI}_{\mathrm{E}}$ data are shown in Fig. $2 \mathrm{~b}$. 


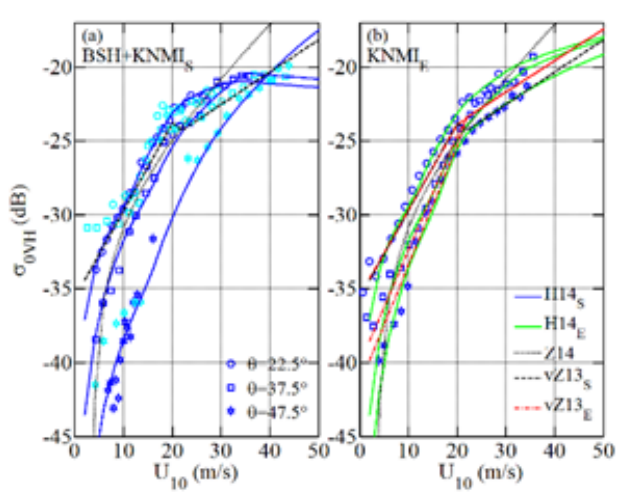

Fig. 2. Comparison of various GMFs and datasets: (a) Combined BSH (dark symbols) and KNMIs (light symbols) datasets and $\mathrm{H} 14_{\mathrm{s}}, \mathrm{Z14}$ and $\mathrm{vZ13} \mathrm{s}_{\mathrm{S}}$ GMFs, and (b) KNMI dataset and H14E, Z14, vZ13s and vZ13E GMFs.

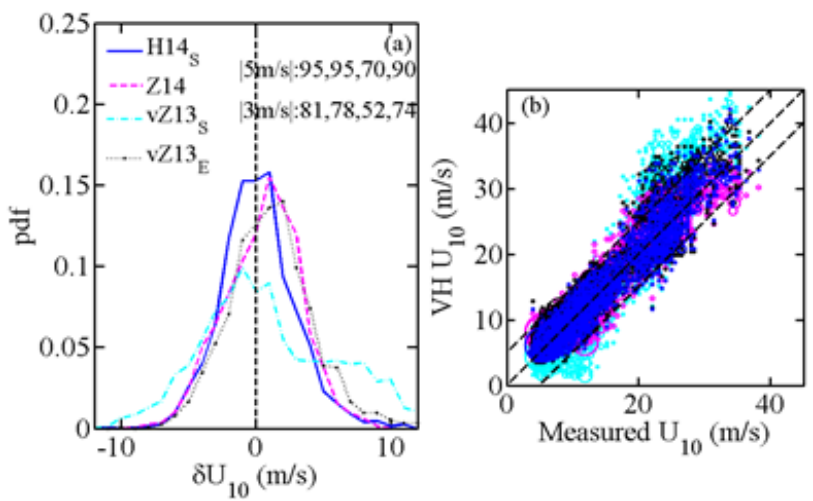

Fig. 3. The wind speed inversion using 4 different GMFs (H14s, Z14, vZ13s and $v Z 13_{\mathrm{E}}$ ): (a) The pdf of $\delta U_{10}$, and (b) the scatter plot of measured and inverted $U_{10}$; BSH dataset.

Figures 3, 4 and 5 show respectively the results of wind speed inversion for the $\mathrm{BSH}, \mathrm{KNMI}_{\mathrm{S}}$ and $\mathrm{KNMI}_{\mathrm{E}}$ datasets using the various GMFs. For each figure, the pdf of the wind speed difference $\delta U_{10}$ (inverted minus measured) is displayed in panel (a) and the scatter plot of inverted vs. measured $U_{10}$ is shown in panel (b) with the color scheme identical to that used in (a). The better performance of the $\mathrm{H} 14$ algorithm is manifested in the narrower distribution of $\delta U_{10}$ near $0 \mathrm{~m} / \mathrm{s}$ (panel (a)) and more compact cluttering of data about the 1:1 diagonal line (panel (b)). The percentage of velocity difference for $\left(\mathrm{H} 14_{\mathrm{s}}, \mathrm{Z14}, \mathrm{vZ13} \mathrm{s}, \mathrm{vZ13_{ \textrm {E } }}\right)$ is $(95$, $95,70,90)$ within $5 \mathrm{~m} / \mathrm{s},(81,78,52,74)$ within $3 \mathrm{~m} / \mathrm{s}$ for the BSH dataset; $(75,69,57,69)$ within $5 \mathrm{~m} / \mathrm{s},(53,52,36$, 49) within $3 \mathrm{~m} / \mathrm{s}$ for the $\mathrm{KNMI}_{\mathrm{S}}$ dataset; and $(97,91,75,94)$ within $5 \mathrm{~m} / \mathrm{s},(87,72,55,84)$ within $3 \mathrm{~m} / \mathrm{s}$ for the $\mathrm{KNMI}_{\mathrm{E}}$ dataset. (2015).

More results and discussions are given in Hwang et al.

\section{SUMMARY}

In this paper, we present a framework to construct the C-band VH GMF. The method is built upon the piecewise

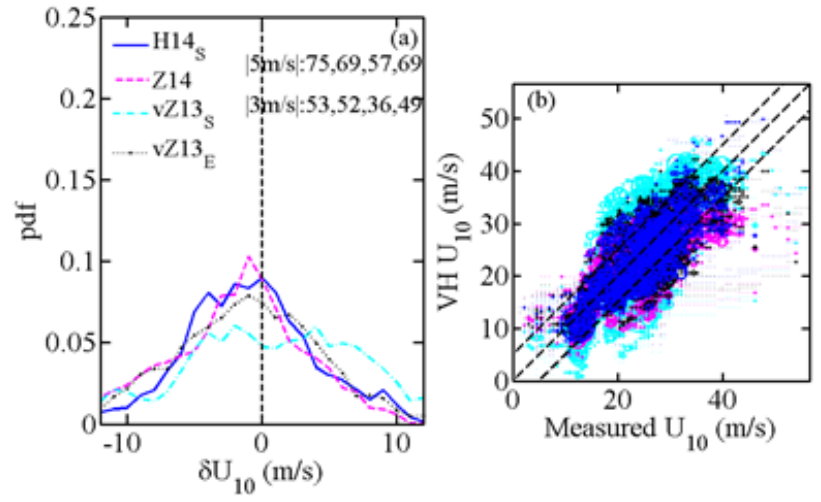

Fig. 4. The wind speed inversion using 4 different GMFs $(\mathrm{H} 14 \mathrm{~s}$, Z14, vZ13s and vZ13E): (a) The pdf of $\delta U_{10}$, and (b) the scatter plot of measured and inverted $U_{10}$; KNMIs dataset.

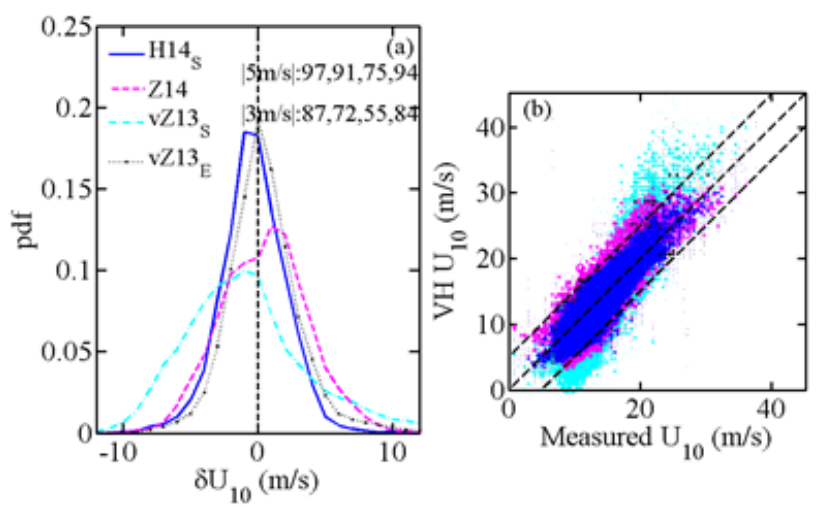

Fig. 5. The wind speed inversion using 4 different GMFs $(\mathrm{H} 14 \mathrm{~s}$, Z14, vZ13s and vZ13E): (a) The pdf of $\delta U_{10}$, and (b) the scatter plot of measured and inverted $U_{10}$; KNMIE dataset.

power-law relationship between $\sigma_{0 V H}(\theta)$ and $U_{10}$. This is consistent with the property of piecewise power-law wind speed relationship of the short scale wind-generated waves serving as the Bragg resonance roughness elements of microwave scattering from the ocean surface (e.g., Hwang and Wang, 2004; Hwang et al., 2013).

Recognizing the difficulty of assembling sufficient collocated and simultaneous radar backscattering and wind velocity measurements to cover a broad range of wind speed and incidence angle, the GMF is designed to work with partial knowledge of the power-law function relating $\sigma_{0 V H}(\theta)$ and $U_{10}$. In particular, the main input parameters are the exponents of the power-law functions in several IABs and WSGs, and the proportionality coefficient for the lowest WSG, of which the most abundant data is expected. It is also designed for easy refinement as new data becomes available or when datasets with different characteristics require special GMF variations.

\section{REFERENCES}

Belmonte Rivas, M., A. Stoffelen, and G.-J. van Zadelhoff: The benefit of $\mathrm{HH}$ and $\mathrm{VV}$ polarizations in retrieving extreme wind speeds for an ASCAT-type scatterometer, IEEE Trans. Geos. Remote Sensing, 52, 7, 4273-4280, 2014. 
Hwang, P. A., B. Zhang, and W. Perrie: Depolarized radar return for breaking wave measurement and hurricane wind retrieval. Geophys. Res. Lett., 37, L01604, doi:10.1029/2009GL041780, 2010a.

Hwang, P. A., B. Zhang, J. V. Toporkov, and W. Perrie: Comparison of composite Bragg theory and quad-polarization radar backscatter from RADARSAT-2: with applications to wave breaking and high wind retrieval, J. Geophys. Res., 115, C08019, doi:10.1029/2009JC005995, 2010b.

Hwang, P. A., W. Perrie, and B. Zhang, Cross polarization radar backscattering from the ocean surface and its dependence on wind velocity. IEEE Geos. Rem. Sens. Lett., 21, 2188-2192, 2014.

Hwang, P. A., A. Stoffelen, G.-J. van Zadelhoff, W. Perrie, B. Zhang, H. Li, and H. Shen, Cross polarization geophysical model function for C-band radar backscattering from the ocean surface and wind speed retrieval. J. Geophys. Res., 120, 893909, doi:10.1029/2014JC010439, 2015.

Lin, C.-C., M. Betto, M. B. Rivas, A. Stoffelen, and J. de Kloe, EPS-SG Wind scatterometer concept trade-offs and wind retrieval performance assessment, IEEE Trans. Geos. Remote
Sens., 50, 2458-2472, 2012.

Vachon, P. W., and J. Wolfe: C-band cross-polarization wind speed retrieval, IEEE Geos. Remote Sensing Lett., 8, 456-458, 2011.

van Zadelhoff, G.-J., A. Stoffelen, P. W. Vachon, J. Wolfe, J. Horstmann, and M. Belmonte Rivas, Scatterometer hurricane wind speed retrievals using cross polarization, Atmos. Measure. Tech. Discuss., 6, 7945-7984, 2013.

Van Zadelhoff, G.-J., Stoffelen, A., Vachon, P. W., Wolfe, J., Horstmann, J. and Belmonte Rivas, M.: Retrieving hurricane wind speeds using cross polarization C-band measurements. Atmos. Measure. Tech., 7, 2, 437-449, 2014.

Zhang, B., W. Perrie, and Y. He: Wind speed retrieval from RADARSAT-2 quad-polarization images using a new polarization ratio model, J. Geophys. Res., 116, C08008, doi:10.1029/2010JC006522, 2011.

Zhang, B., W. Perrie, J. A. Zhang, E. W. Uhlhorn, and Y. He: High resolution hurricane vector winds from C-band dualpolarization SAR observations, J. Atmos. Oceanic Tech., 31, 272-286, 2014. 